\title{
Reconstruction of Chronic Subtalar Dislocation
}

\author{
MC Chan ${ }^{1}$, Suheal A Khan ${ }^{2}$, Jeffrey GM Tan ${ }^{3}$
}

\begin{abstract}
Introduction: Subtalar dislocations are a rare injury, comprising 1-2\% of all large joint dislocations and $15 \%$ of all peri-talar injuries. Most subtalar dislocations are managed acutely, with the documented reconstruction of chronic dislocations rare. This case report aims to present our experience with the first documented use of an Ilizarov frame for bony and soft tissue reconstruction in a case of chronic subtalar dislocation. Materials and methods: A single patient surgically treated for chronic subtalar dislocation was followed over a 2-year period, and retrospectively reviewed.

Results: A 67-year-old lady presented with an over 10-year history of worsening ankle pain and significant varus deformity with skin contractures, limiting ambulation over the past 6 months. Computed tomography confirmed medial dislocations of the subtalar and talonavicular joints with head and neck fractures of the talus and generalized osteoarthritis. A two-stage reconstruction involving an llizarov frame and subsequent arthrodesis allowed for correction of soft tissue and bone with the return of independent ambulation.

Conclusion: Although the correction of bony deformities is important in reconstruction, soft tissue management is crucial and must not be overlooked. An llizarov frame is ideal for correction of both the bony deformity and soft tissue contractures. Arthrodesis can be performed when the overlying soft tissue is able to accommodate correction of the varus deformity and reduction of the dislocations. While an llizarov frame requires both an experienced surgeon and a motivated patient, in the right hands, good functional recovery can be returned.

Keywords: Chronic, Dislocation, Ilizarov, Reconstruction, Subtalar.

Strategies in Trauma and Limb Reconstruction (2019): 10.5005/jp-journals-10080-1412
\end{abstract}

\section{INTRODUCTION}

Subtalar dislocations are a rare injury, comprising approximately $1-2 \%$ of all dislocations. ${ }^{1}$ Broca's classification in $1853^{2}$ initially described medial (80-85\%), lateral (15-20\%), and posterior $(2.5 \%)$, with anterior $(1.5 \%)^{3}$ dislocations added to the classification by Malgaigne and Buerger. ${ }^{4}$

Most subtalar dislocations are managed in the acute setting, with documented management of chronic injuries rare, likely due to the uncommon yet debilitating nature of the injury. A single publication by Bajuri et al. ${ }^{5}$ in 2013 described such a case in a 57-year-old lady with a history of chronic dislocation neglected for over 10 years. Her isolated dislocation of the talo-calcaneal joint associated with a nonunited talar fracture and osteoarthritic talo-crural joint was managed with a single-stage arthrodesis, allowing for the return of ambulation.

This case report aims to present our experience with a different approach to bony and soft tissue reconstruction in a case of chronic subtalar dislocation neglected for over 10 years.

\section{Case History}

A 67-year-old Chinese lady with a past medical history of hypertension was referred by her primary physician to the specialist orthopedic clinic with a 6-month history of worsening pain in her left ankle and difficulty in ambulation. She reported an inversion injury to the left ankle over 10 years ago, but at the time declined surgical intervention. She has since had worsening chronic deformity of the left foot and the ankle, but remained able to ambulate independently until 6 months prior to presentation. She had sought alternative medical therapy, including acupuncture, over the past 6 months, but with no improvement.

On examination, she walked with an antalgic gait. There was a marked varus deformity of her left foot and ankle, but there was no neurovascular deficit. Importantly, it was noted that the severity of varus deformity had resulted in marked tension of the skin overlying
${ }^{1}$ Ministry of Health Holdings, Singapore

${ }^{2}$ Mobile International Surgical Teams (MIST) Foundation, Manchester, UK

${ }^{3}$ Department of Orthopaedic Surgery, Khoo Teck Puat Hospital, Singapore

Corresponding Author: MC Chan, Ministry of Health Holdings, Singapore, Phone: +65 9828 9877, e-mail: mingchunchan@hotmail.com How to cite this article: Chan MC, Khan SA, Tan JGM. Reconstruction of Chronic Subtalar Dislocation. Strategies Trauma Limb Reconstr 2019;14(1):53-56.

Source of support: Nil

Conflict of interest: None

the lateral malleolus, with contractures over the medial side of the ankle. In addition to medial soft tissue contractures and lateral skin tension from chronicity of the deformity, skin atrophy and asteatotic eczema associated with her advanced age made soft tissue a major concern (Fig. 1).

Plain radiographs showed gross sclerosis and disorganization of the bones in the foot and ankle, with medial dislocation of the tarsus and distal foot, as well as associated dislocation of the calcaneum and cuboid (Fig. 2).

Management options were discussed and the patient was keen on surgical reconstruction in view of worsening deformity and pain affecting premorbidly independent ambulation and daily function.

Computed tomography $(\mathrm{CT})$ with $3 \mathrm{D}$ reconstruction imaging was then performed preoperatively. Findings supported earlier plain radiographs, with medial dislocations of the subtalar and talonavicular joints. The talus was deformed with bony loss and fragmentation of the head and the neck. Pseudoarticulations between the calcaneum and tibia had developed with diffuse osteoarthritic changes involving the mid-tarsal joint (Figs 3 and 4).

In view of the chronicity and severity of both the bony deformities and the soft tissue and skin contractures, a two-staged 


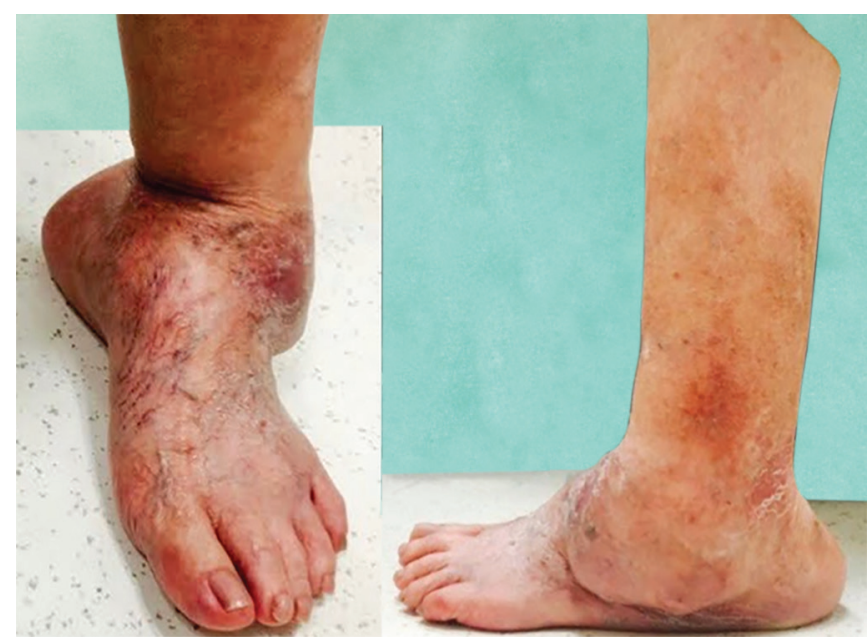

Fig. 1: Clinical pictures of foot and ankle on presentation: anteriorposterior (AP) (left) and lateral (right) views
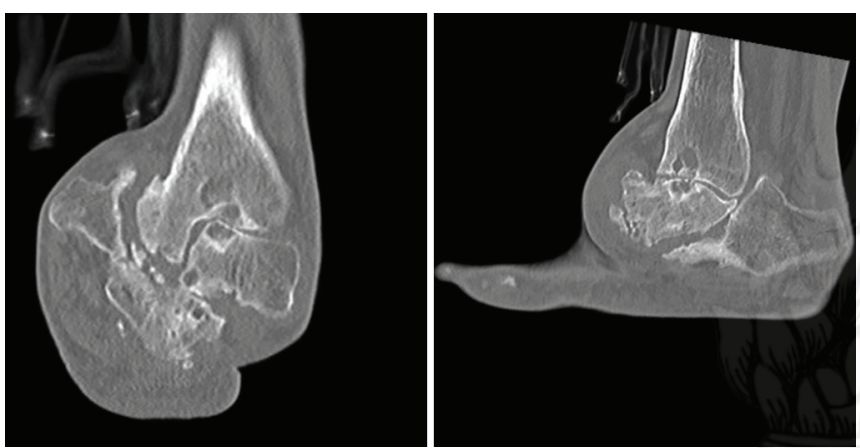

Fig. 3: Preoperative CT: frontal (left) and parasagittal (right) planes

procedure was planned with the goal of returning pain-free weight bearing and ambulation to the patient. This involved an initial application of an Ilizarov external fixator, followed by subsequent tibio-talo-calcaneal fusion with a hindfoot arthrodesis nail.

The first stage of the operation was the application of an llizarov frame with a foot-plate, using the TL-Hex (Truelok Hexapod System, Orthofix, Italy). The purpose of the llizarov frame was two fold: first, to reduce and stabilize the bony deformities; second, and, crucially, to allow gradual stretching of the soft tissue and skin. Intraoperatively two smooth wires and one olive wire were inserted into the calcaneum with an anterior forefoot smooth wire inserted through the metatarsal shafts. The foot frame was applied and aligned to the calcaneum and forefoot. Further rings were then applied in the tibia
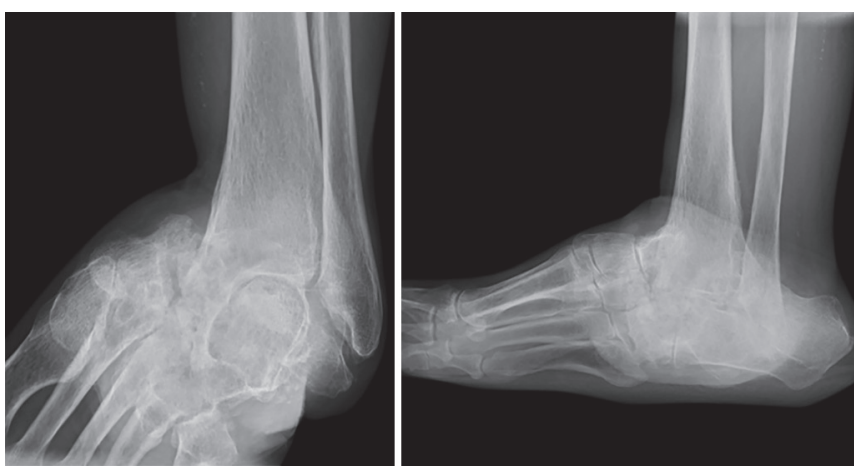

Fig. 2: Preoperative plain radiographs: AP (left) and lateral (right) views

and secured. Postoperatively, she was kept nonweight-bearing for 2 months, with postoperative care in a rehabilitative facility. Gradual correction of the deformity was done by the patient, by turning the struts on her TL-Hex frame daily following a protocol generated by the TL-Hex web-based program (Figs 5 and 6).

The external fixator was removed 8 weeks later and the patient was placed in a cast for 2 weeks. This allowed for healing of the pin sites before the second stage of reconstruction. She was allowed to fully weight-bear as tolerated in the cast and continued with physiotherapy. Ten weeks after the initial operation, she underwent arthrodesis of the hindfoot and talonavicular fusion with percutaneous release of the tendo-Achilles. A lateral incision allowed for a distal fibulectomy. The tibiotalar, talonavicular, and subtalar joints were prepared and reduced with the foot in plantigrade and slight valgus. The calcaneum and the talus were then nailed and fused through a heel incision with bone grafts (taken from morselization of the removed fibula) added to the tibiotalar, subtalar, and talonavicular joints. No implants were used for fixation of the talonavicular joint as the reduction was stable, partly contributed to by surrounding soft tissue contractures. Postoperatively, she was placed in a back-slab and initially started on a nonweight bearing program on crutches with the physiotherapists (Fig. 7).

Ten days postoperatively, she was noted in the outpatient specialist orthopedic clinic to have localized signs of superficial, necrotic skin infection over the lateral incision site for which she was admitted for a 7-day course of intravenous antibiotics to which the infection responded well. Her weight-bearing status was escalated to partial weight bearing after 9 weeks and full weight bearing within 12 weeks postoperatively.

The patient achieved her goal of independent weight bearing and ambulation 7 months following the start of her two-stage

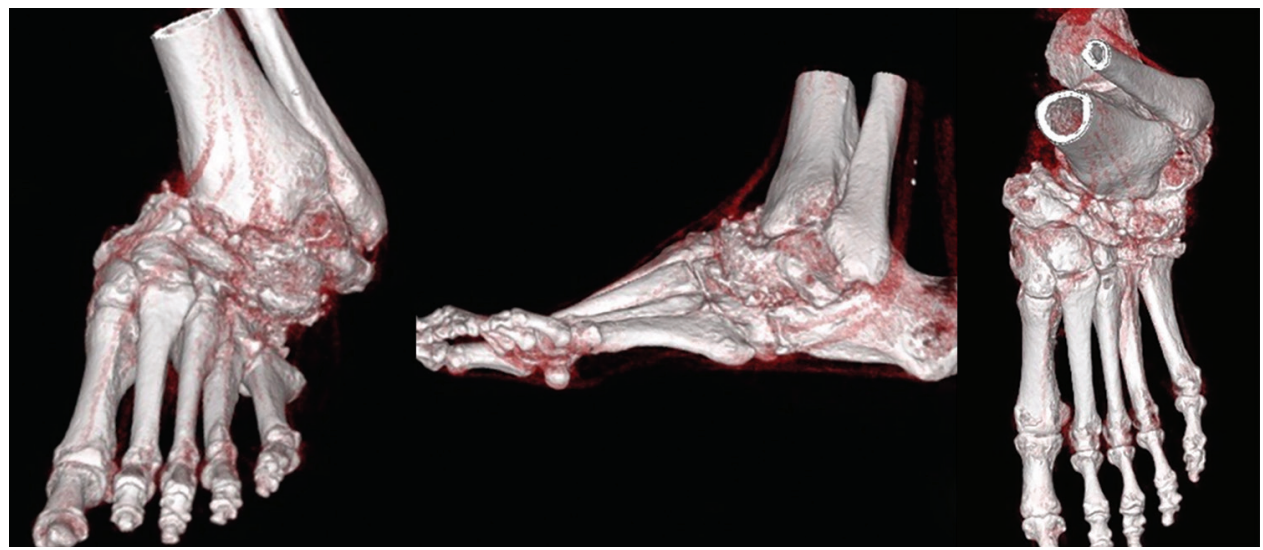

Fig. 4: CT 3D reconstruction: AP (left), lateral (middle), and axial view (right) 


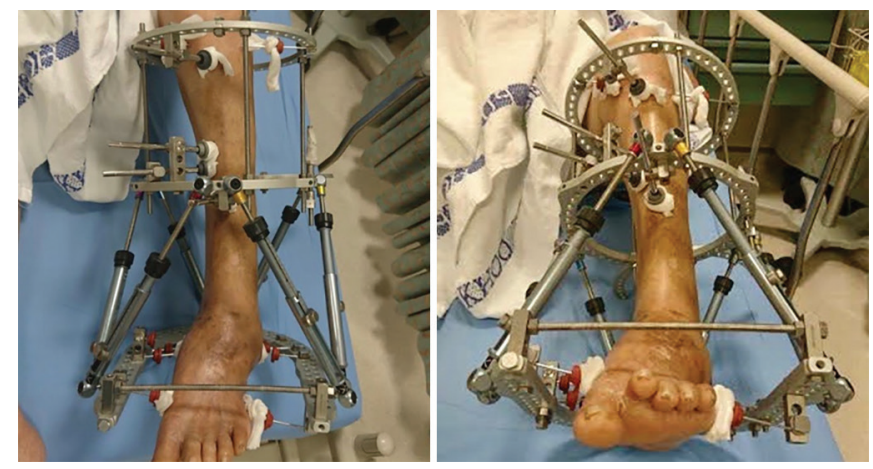

Fig. 5: Clinical pictures of foot and ankle post-operatively with TL-Hex frame

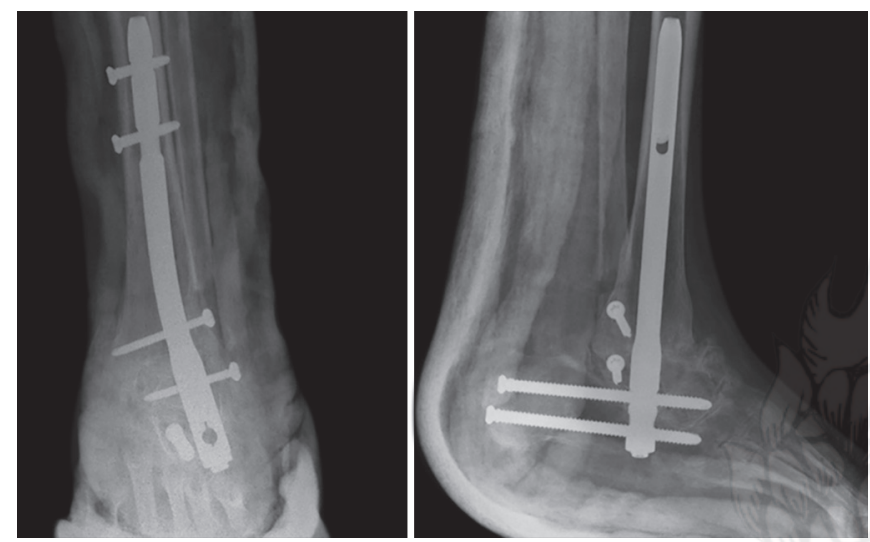

Fig. 7: Initial postoperative plain radiographs following arthrodesis, fusion, and fibulectomy: AP (left) and lateral (right) views

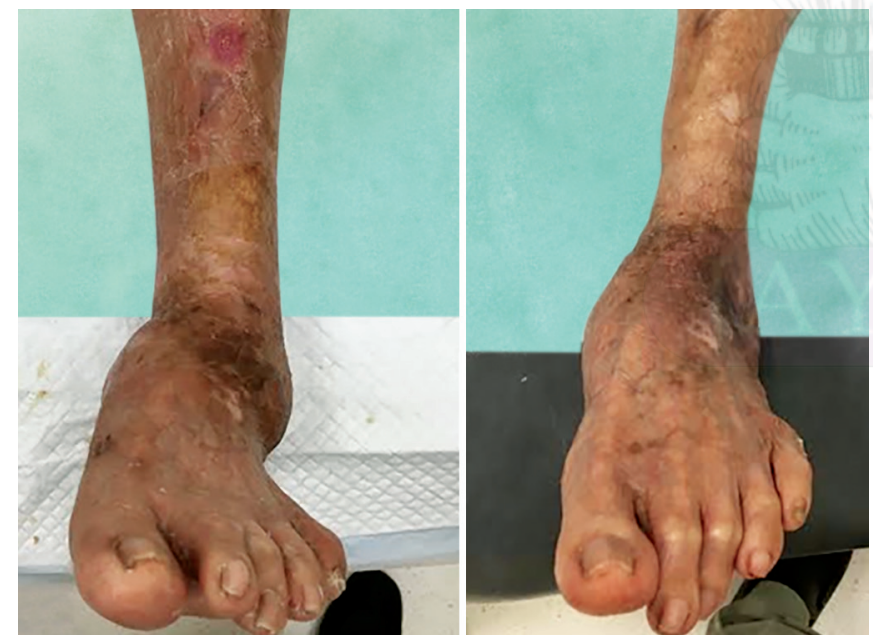

Fig. 9: Clinical pictures comparing foot and ankle preoperatively (left) and postreconstruction (right)

reconstructive process, with good cosmetic and functional stability in her foot and ankle. A final day surgery procedure to remove a prominent distal interlocking screw in the lateral calcaneal tuberosity was performed 5 months following arthrodesis but the patient remained happy with the result (Figs 8 to 10).

\section{Discussion}

The treatment of subtalar dislocations occurs in the acute setting commonly as the injury is associated with high-energy trauma and

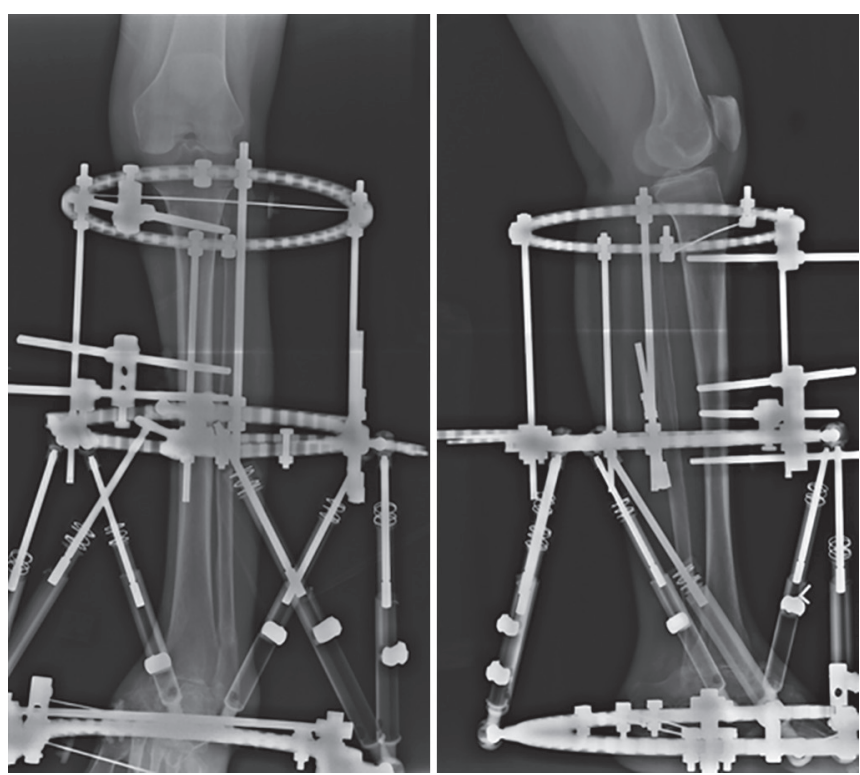

Fig. 6: Postoperative plain radiographs with TL-Hex frame: AP (left) and lateral (right) views
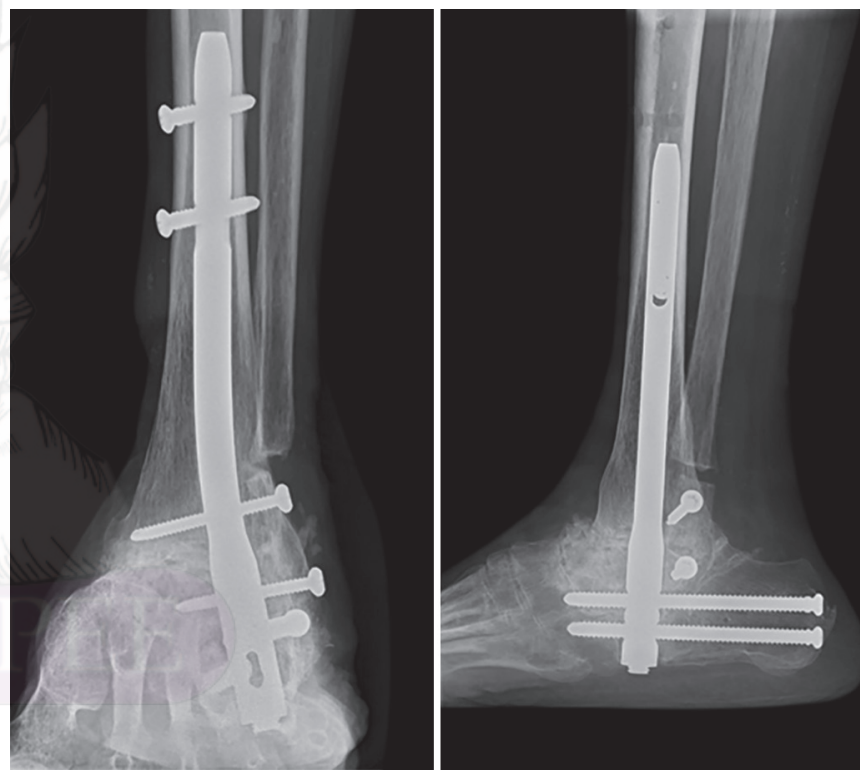

Fig. 8: Plain radiographs 6 month following start of two-stage reconstruction: AP (left) and lateral (right) views

produces significant deformity and functional deficit. Considering the complexity of her injuries, it is likely the patient suffered a medial dislocation of the subtalar and talonavicular joints and possible associated fractures of the talus. Continuous weight bearing of the joint probably worsened the deformities with the development of osteoarthritis.

Two surgical options were considered. The first option was a single-stage reconstruction involving arthrodesis, with options such as a pantalar fusion considered, the main benefit of which was the minimization of the number of operative procedures and anesthetic risks. The second option was a two-stage reconstruction which first involved the application of an llizarov external fixator. The llizarov frame reduces the dislocations gradually and, crucially, allows for stretching of the soft tissues and skin. The second stage involved the removal of the frame and 

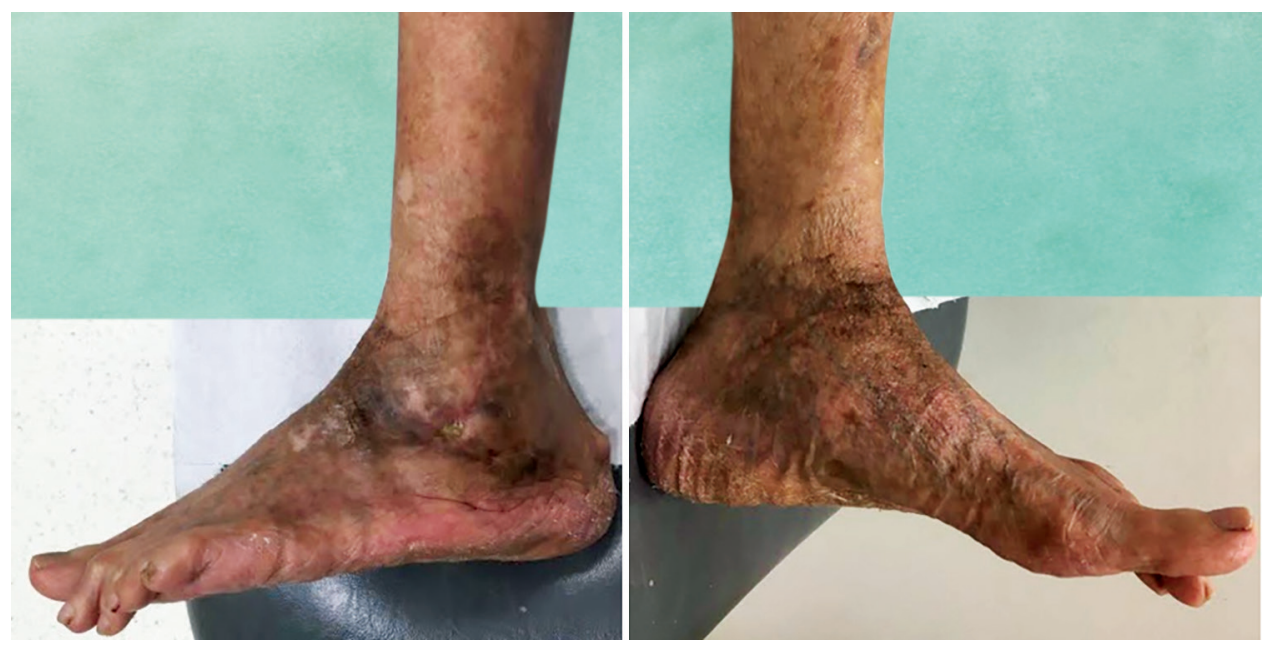

Fig. 10: Clinical pictures of reconstructed foot and ankle: lateral (left) and medial (right) views

arthrodesis as well as bone grafting for long-term stability and fixation. A tibio-talo-calcaneal fusion with a hindfoot arthrodesis nail was sufficient as the talus and the navicular was found to be stable, compressed, and locked in place after hindfoot nailing to fuse the tibio-talo-calcaneal joints.

The options were discussed with the patient and, given the severity of her soft tissue contractures as well as her personal motivation and family support, she was agreeable to the twostage reconstruction. Patient compliance in the llizarov frame was a significant concern due to the need for diligent daily pin site care and frame adjustment. The success of the frame and correction of the soft tissues as well as bony deformities was in due to the diligence of the patient and family support. Common complications such as pin site infections are a genuine concern and the patient was monitored closely in the outpatient setting.

This was a successful case of a two-stage and dual modality (external fixation followed by internal fixation) reconstruction of significant foot deformity. The llizarov method of gradual correction was ideal for addressing both the bony deformity and soft tissue contractures. However, it requires a motivated patient and close follow-up to manage potential complications. Subsequent arthrodesis can then be performed when the overlying soft tissue has adapted to the corrected foot position. The llizarov method requires both an experienced surgeon and a motivated patient but, in the right hands and for the appropriate cases, good functional recovery can be achieved.

\section{Ethical Approval}

The study was approved by the research ethics committee of the institution.

\section{Informed Consent}

Proper informed consent was taken and patient was explained about the procedure before starting treatment.

\section{References}

1. Prada-Cañizares A, Auñón-Martín I, et al. Subtalar discloation: management and prognosis for an uncommon orthopaedic condition. International Orthopaedics 2016;40:999-1007. DOI: 10.1007/s00264-015-2910-8.

2. Broca P. Memories sur les luxations sous-astragaliennes. Mem Soc Chir 1853;3:566-656.

3. Horning J, DiPreta J. Subtalar dislocation. Orthopedics 2009;32(12):904-908. DOI: 10.3928/01477447-20091020-17.

4. Malgaigne JF, Buerger CG. Die Knochenbrüche und Verrekungen. Stuttgart: Rieger; 1856. p. 820.

5. Bajuri MY, Johan RR, et al. Neglected subtalar dislocation with unstable foot; to walk away or step our foot in? BMJ Case Rep 2013. DOI: $10.1136 / \mathrm{bcr}-2012-007697$. 